\title{
Factors influencing the academic performance of the female medical students in preclinical and clinical years
}

\author{
*Sarah M.S. Shathele ${ }^{1}$, Anitha Oommen ${ }^{2}$ \\ ${ }^{1}$ Medical Student, Faculty of Medicine, Northern Border University, Arar, Saudi Arabia \\ ${ }^{2}$ Department of Anatomy, Northern Border University, Arar, Saudi Arabia \\ ${ }^{*}$ Corresponding author's email: alfadlelmula@yahoo.com
}

\begin{abstract}
The present study was undertaken to investigate the factors influencing the academic performance of the female medical students in the preclinical $\left(2^{\text {td }}\right.$ and $3^{\text {rd }}$ year) and clinical years $\left(4^{\text {th }}\right.$ and $5^{\text {th }}$ years $)$.A crosssectional study was carried out involving 100 female medical students (preclinical and clinical) in Northern Border University. A comprehensive questionnaire was used to evaluate the factors influencing their academic performance. A comparison was done between the factors influencing the academic performance of the preclinical and the clinical students. The strongest positive factors which influenced the academic performance of all the female medical students were the facility available for study, family support, and awareness about the medical course. Anxiety and stress and lack of sleep were the strong negative factors in both. There was significant difference between preclinical and clinical students regarding the time for self-study $(p<0.05)$ and lack of sleep $(p<0.05)$. Although the strongest positive factors and negative factors were similar in both preclinical and clinical students, there was variation in factors like English skills, personal time, family time and time for self-study. There was an increase in the English language skills, competition in studies and time for self-study in the clinical students when compared to the preclinical students, though the difference was not statistically significant.
\end{abstract}

Keywords: Medical students, Female, Academic performance, Facilities, Anxiety, Stress, Saudi Arabia.

\section{INTRODUCTION}

Many studies have been done in different parts of the world to find out the difficulties faced by medical students. Studies have also been done in different parts of Saudi Arabia to assess the academic performance of undergraduate medical students (Al-Mulhim et al., 2012). Researchers have also looked into the sociodemographic factors influencing the student performance in a Saudi medical school (Salem et al., 2013). The difficulties faced by first year medical students were investigated in Umm Alqura University in Saudi Arabia (Almoallim et al., 2010). The impact of the gender of the student and English language skills on the academic performance of the medical students has been studied by the researchers of King Saud University which showed that the female medical students were superior in academic performance when compared to male students in the preclinical years (Al-Mously et al., 2013).

However they reported that the English language skills was the significant predictor of academic performance rather than gender. Since the proportion of female medical students is increasing in medical schools with time, there is concern regarding their differing performance in examinations (Sawair et al., 2009; Hinkley,1997; Hall et al., 2001). Since the medium of communication in the medical schools is English, the Arabic speaking students find English as a challenge in learning the medical subjects. The present study was therefore undertaken to find out the different factors influencing positively and negatively the academic performance of the female medical students in the preclinical and clinical years in the Northern Border University. Therefore, the present study aimed to: 1investigate the positive and negative factors which influence the academic performance of the preclinical and clinical female students, and 2- compare the differences in the factors, if any, which influence the academic performance of the preclinical students and the clinical students. 


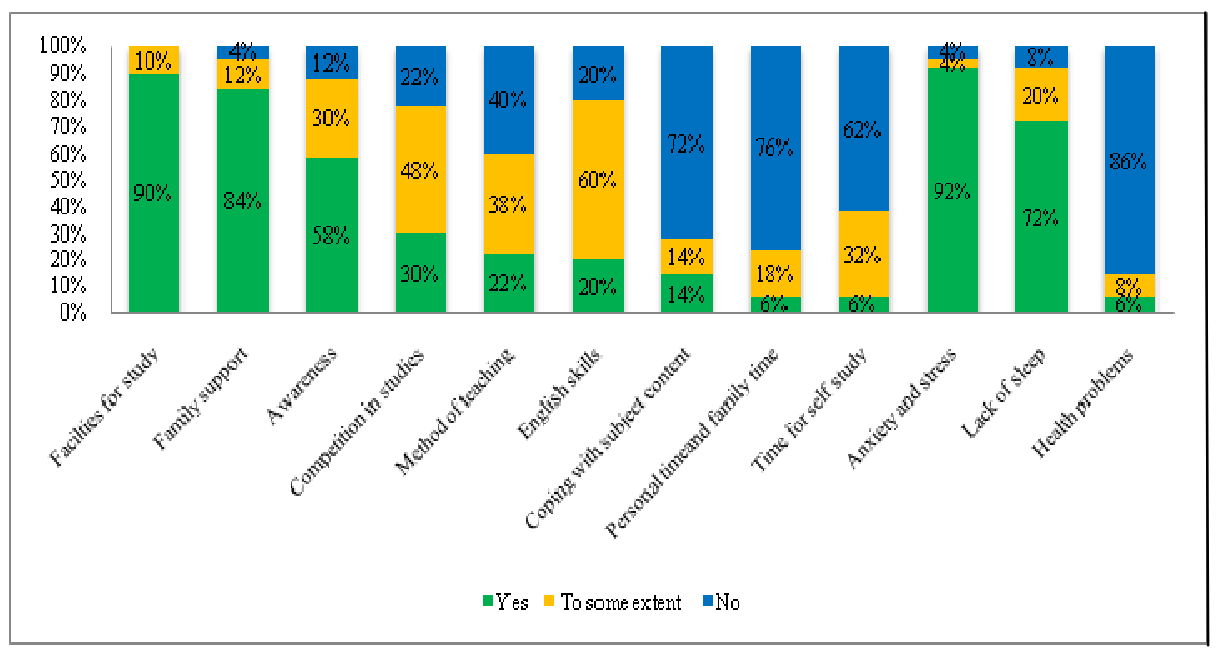

Figure 1: Factors influencing performance in the preclinical group of female medical students in the Northern Border University

\section{METHODS}

\section{Venue of study}

A cross sectional study was carried out in the Northern Border University among 100 female students who were in the $2^{\text {nd }}-5^{\text {th }}$ years of medical school.

\section{Subjects}

25 students from each year were selected. The students selected for the study were volunteer participants to whom the purpose of the study was explained.

\section{Data collection}

A comprehensive questionnaire which contained questions like the facilities for study, English language skills, teaching methodology, health problems and social factors was given to the students to assess the factors influencing the academic performance. The questions were written in Arabic and English. In the questionnaire given to the clinical students, the marital status and the clinical skills were added as additional factors. The responses expected from the students were "yes", "to some extent" and "no". After clarifying doubts regarding the questionnaire, the students were asked to complete the questionnaire within 15 minutes and it was collected by the researcher.

\section{Data analysis}

The data was analyzed using SPSS software. Chi-square test was used to find out possible significant differences between factors influencing students' performance in the preclinical and clinical years. The degree of statistical significance was fixed as $p<0.05$.

\section{RESULTS}

The analysis of factors influencing students' academic performance as perceived by students themselves are summarized and presented in Figure 1 and Figure 2. Figure 1 shows the factors affecting the academic performance of the preclinical students whereas Figure 2 demonstrates the factors affecting the academic performance of the clinical students.

Both in the preclinical and clinical years the positive factors contributing to the academic performance of the female medical students were facilities for study, family support and awareness about the medical course. Competition in studies was more in the clinical students (42\%) when compared to preclinical students (30\%), the difference, though, was not statistically significant. The English language skills were higher in the clinical students $(36 \%)$ when compared to the preclinical students (20\%) which was also not statistically significant. Only $22 \%$ of preclinical students and $26 \%$ of the clinical students were of the opinion that the teaching methodology was good. As far as the subject content was concerned, only $14 \%$ of both preclinical and clinical students could cope with the subject content. $6 \%$ of the preclinical students were of the opinion that the personal time and family time was sufficient, while $0 \%$ of the clinical students had the opinion that the personal time and family time was sufficient.

$30 \%$ of the preclinical students showed a competitive attitude in studies while $42 \%$ of the clinical students had a competitive attitude towards studies. The health problems 


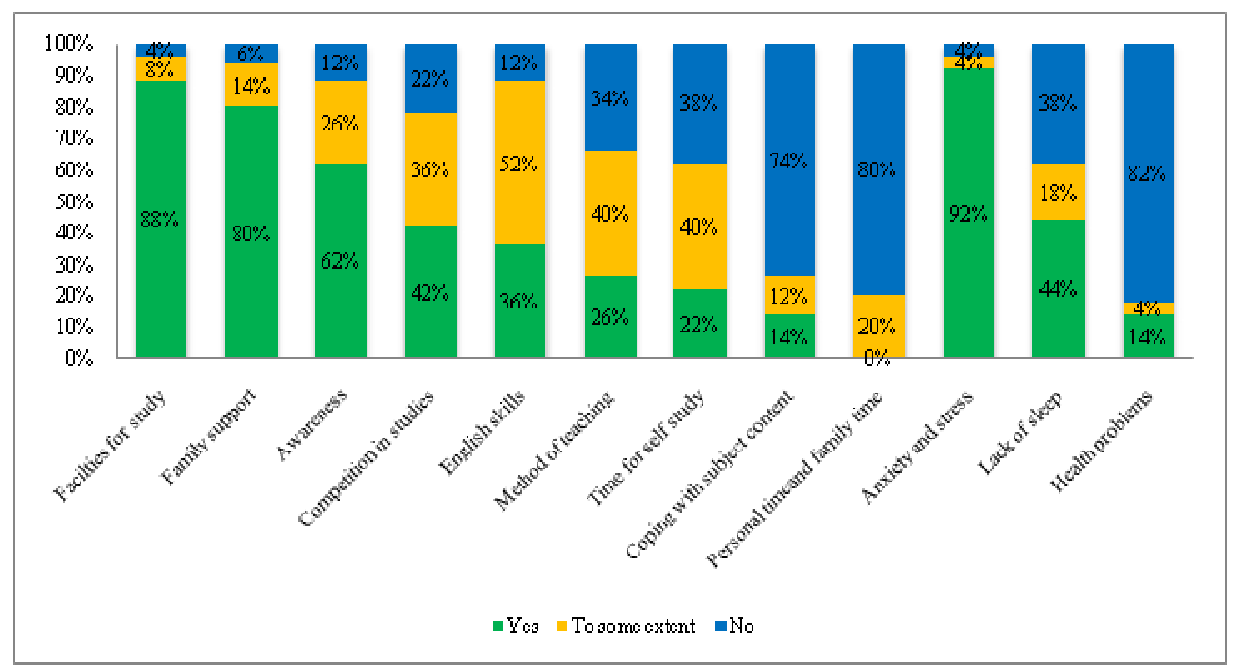

Figure 2: Factors influencing performance in the clinical group of female medical students in the Northern Border University

Table 1. Availability of time for self-study in the preclinical and clinical group of female medical students in the Northern Border University

\begin{tabular}{|l|c|c|c|c|}
\hline \multirow{2}{*}{ Group } & \multicolumn{3}{|c|}{ Time for self-study } & \multirow{2}{*}{ Total } \\
\cline { 2 - 4 } & Yes & No & To some extent & $50(100 \%)$ \\
\hline Preclinical & $3(6 \%)$ & $31(62 \%)$ & $16(32 \%)$ & $50(100 \%)$ \\
\hline Clinical & $11(22 \%)$ & $19(38 \%)$ & $20(40 \%)$ & $100(100 \%)$ \\
\hline Both & $14(14 \%)$ & $50(50 \%)$ & $36(36 \%)$ & \\
\hline
\end{tabular}

Table 2. Lack of sleep complaint in the preclinical and clinical group of female medical students in the Northern Border University

\begin{tabular}{|l|c|c|c|c|}
\hline \multirow{2}{*}{ Group } & \multicolumn{3}{|c|}{ Lack of sleep } & \multirow{2}{*}{ Total } \\
\cline { 2 - 4 } & Yes & No & To some extent & $50(100 \%)$ \\
\hline Preclinical & $36(72 \%)$ & $4(8 \%)$ & $10(20 \%)$ & $50(100 \%)$ \\
\hline Clinical & $22(44 \%)$ & $19(38 \%)$ & $9(18 \%)$ & $100(100 \%)$ \\
\hline Both & $58(58 \%)$ & $23(23 \%)$ & $19(19 \%)$ & \\
\hline
\end{tabular}

were more in the clinical students (14\%) when compared to preclinical students (6\%). The level of anxiety and stress was very high and the percentage was the same in both preclinical and clinical students (92\%).

There was a statistically significant difference between preclinical and clinical group of students only in two factors, which were "time for self-study" and "lack of sleep" as shown on Tables 1 and 2.

$62 \%$ of preclinical students indicated that they did not find time for self-study whileonly $38 \%$ of clinical students said that they did not find time for self-study. The chisquare test showed a p-value $<0.05$ indicating that there was a statistically significant difference between preclinical and clinical students. (Table1).

$72 \%$ of preclinical students indicated that they had lack of sleep whileonly $44 \%$ of clinical students said that they had lack of sleep. The chi-square test showed a pvalue $<0.05$ indicating that there was a statistically significant difference between preclinical and clinical students (Table 2). 
Table 3. Improvement of Clinical skills as conceived by female clinical students in the Northern Border University

\begin{tabular}{lcc}
\hline Response & Count & Percent \\
\hline Yes & 30 & $60 \%$ \\
No & 2 & $4 \%$ \\
To some extent & 18 & $36 \%$ \\
\hline Total & $\mathbf{5 0}$ & $\mathbf{1 0 0 \%}$ \\
\hline
\end{tabular}

The clinical skills was a factor included in the questionnaire only for the clinical students. $60 \%$ of the students said that their clinical skills have improved while $36 \%$ of students indicated that their clinical skills have improved only to some extent. $4 \%$ of students said that they had no improvement in their clinical skills (Table 3 ).

\section{DISCUSSION}

A challenge in the field of medical education is to identify the factors that can lead to a good academic performance. Even though many studies have been done in different countries, and in different parts of Saudi Arabia, there has been no reported study done in the Northern border province. This study, therefore, has been undertaken to highlight the factors that influence the academic performance of the female medical students of Northern Border University.

The present study displays the views of 100 female Saudi medical students of the preclinical and clinical years in the Northern Border University showed that there are many positive and negative factors which influence the academic performance. The positive factors were "awareness about the medical course", "family support" and "facilities for study". The negative factors were difficulty in speaking and writing in English language (" English language skills"), "inadequate time" for self-study, personal relaxation and interaction with the family.

\section{English language skills}

The English language skills improved in the clinical years though not significantly. $52 \%$ of the clinical students had improved to some extent while $12 \%$ had no improvement at all. This correlated with the findings of a study which reported that the global rating of the unsatisfactory spoken English language fluency was associated with poorer performance in medical communication skills especially during examinations (Chur-Hansen et al., 1997).

\section{Time management}

Effective time management skills are essential for continued academic success. Students who do not plan their time effectively run out of time before they can master the subject content. Therefore providing students with assistance and support in time management should help them to utilize their study time more efficiently and effectively which in turn will improve their academic performance (Abdulghani et al., 2014). In the present study, only $6 \%$ of the preclinical students said that they had adequate time for self-study, while $32 \%$ of students indicated that they had time for self-study to some extent. Among the clinical students, $22 \%$ of the students were of the opinion that they had adequate time for self-study while $40 \%$ of the students said that they had time for selfstudy to some extent. As far as the personal time and family time were concerned, only $6 \%$ of the preclinical students indicated that they had adequate personal time and family time while $18 \%$ of the students said that they had personal time and family time to some extent. $0 \%$ of the clinical students said that they had personal time and family time while $20 \%$ of clinical students indicated that they had personal time and family time to some extent.

\section{Anxiety and stress}

In the present study, anxiety and stress was a major negative factor similar to the studies done by other researchers (Abdulghani et al., 2011; Salgar, 2014;Dyrbyeet al., 2005; Alakhtarand Al-Homaidan, 2014; Frischenschlageret al., 2005). 92\% of the preclinical and clinical students said that they are suffering from anxiety and stress. Students who are entering the medical field already have concerns about medical school in the process of anticipating the necessary adjustment to the challenges ahead of them. Preventive programs should begin early in the medical education to address the wide variety of concerns which are related to academic program and interpersonal relationships (Smith et al.,2007).

\section{Marital status}

In the questionnaire given to the clinical students, a question was also included about the marital status in order to find out whether the marriage affected their 
academic performance as they had added responsibilities after marriage. Out of the 50 female clinical students, 18 students were married. Only 3 married students (16.7\%) said that their studies were affected due to the marriage responsibilities. Two students (11.1\%) indicated that their studies were affected to some extent because they were married. 13 students $(72.2 \%)$ were of the opinion that the marriage as such did not affect their studies. Our findings were similar to the studies done by researchers from King Faisal University, Saudi Arabia (Abou-Elhamd et al., 2014).

\section{Gender}

In the field of medical education, the issue of gender has become increasingly important for academicians, health administrators and society at large. Several studies have found that female undergraduate students perform better than male students in overall scores in basic and clinical subjects. There are many factors which contribute to this like the subject specific performance. Studies have reported that female students perform better in psychiatry, pediatrics and obstetrics and gynecology (Deepak et al., 2011). In the present study, only $14 \%$ of the students both in the preclinical and clinical years said that they could cope with the content of the subjects taught in our medical school. $14 \%$ of the preclinical students and $12 \%$ of the clinical students indicated that they could handle the subject content to some extent.

\section{Teaching methodology}

Many factors influence learning styles such as brain processing, culture and creative thinking. The students use different sensory modalities for assimilating knowledge and information. Four sensory modalities described by Fleming are: visual (V), Aural (A), Reading/writing (R), Kinesthetic (K). Briefly a visual student prefers to learn visually and an aural student prefers to use listening techniques to learn. Some students use reading and writing as their first preferences for assimilating and accommodating information. A kinesthetic student experiences learning by all sensory channels including somato-sensory, auditory, olfactory, gustative and visual (Nuzhat et al., 2011). It has been suggested that teachers need to understand the learning strategies in order to facilitate student learning. In the present study, only $22 \%$ of the preclinical students were of the opinion that the teaching methodology was good, which included sufficient explanation time and use of audio-visual aids. Among the clinical students only $26 \%$ were satisfied with the teaching methodology. This highlights the importance of improving the quality of teaching methodology taking into consideration the learning style preferences of the medical students. Health education programs, mentorship and a reduction in information overload in the curriculum can be important strategies to enable undergraduates cope better with the demands of medical education (Ko et al., 1999).

\section{Health problems}

Health is an important factor in academic performance; $6 \%$ preclinical students and $14 \%$ of clinical students said that they are having health problems. Regular medical check and proper diet advice should be given to the female medical students as it can affect their academic performance.

\section{Lack of sleep}

In a study done among the medical students of King Saud University, Riyadh, Saudi Arabia, a high prevalence of sleep disorder was found among the first, second and third year medical students. This study showed that there was more sleep disorders in female students than male students (Abdulghani et al., 2012). In the present study, $72 \%$ of preclinical female medical students and $44 \%$ of female clinical students said that they did not have adequate sleeping time. This can affect their academic performance together with physical and psychological well-being.

\section{CONCLUSION}

The main factors which affect the academic performance of the female medical students during the preclinical and clinical years in the Northern Border University are insufficient time for self-study, subject overload, inadequate proficiency in English language, insufficient sleeping time, anxiety and stress, teaching methodology and health problems. There was a significant difference between the preclinical students and clinical students in relation to the time for self-studyand sleep pattern. The preclinical students had shorter time for self-study and sleep which can strongly affect their academic performance and their future career as the basic medical subjects are taught during the preclinical years.

\section{RECOMMENDATIONS}

1. Teaching English language along with medical terminology in the preparatory year.

2. Revision of the preclinical syllabus to include subject content relevant for undergraduate medical education.

3. Adjustment of time frame allotted for medical subjects to help the students cope with the subject content.

4. Strict adherence to the medium of communication as English in undergraduate teaching. 
5. Spacing of examinations to give students adequate time to prepare for the examinations.

6. Giving advice to the students regarding time management.

7. Introduction of creative methods of teaching to make the subjects interesting to the students e.g. Group assignments and small video clippings.

8. Have medical check during the course at least once every 6 months.

9. Involving them in health awareness programs and rural medical camps in the clinical years.

\section{ACKNOWLEDGMENTS}

We are grateful to the female medical students of $2^{\text {nd }}-5^{\text {th }}$ year for giving their valuable time to give an honest feedback regarding the factors that influence their academic performance. We are thankful to the student research wing of Northern Border University for approving our research project thereby encouraging a female medical student to do this research study. We also would like to express our gratitude to Dr. Zakariya Mohammed who helped with the statistical analysis.

\section{REFERENCES}

Abdulghani HM, Al-Drees AA, Khalil MS, Ahmad F, Ponnamperuma GG, Amin Z (2014). What factors determine academic achievement in high achieving undergraduate medical students? A qualitative study. Medical teacher. 36:S43-S48.

Abdulghani HM, Alkanhal AA, Mahmoud ES, Ponnamperuma GG, Alfaris EA (2011). Stress and its Effects on Medical students: A Cross-sectional Study at a College of Medicine in Saudi Arabia. Journal of Health Population and Nutrition. 29:516-522.

Abdulghani HM, Alrowais NA, Bin-Saad NS, Al-Subaie NM, Haji AMA, Alhaqwi Al (2012). Sleep disorder among medical students: Relationship to their academic performance. Medical teacher. 34:37-41.

Abou-Elhamd KEA, Al-Wadaani HA, Al-Mulhim ARS (2014). Female medical undergraduate; does marriage affect the academic performance in a Saudi University? Professional Med J. 21(3):566569.

Alakhtar AM, Al-Homaidan HT (2014). Depression among medical students at Qassim University rate, severity, and contributing factors; using BDI 11. IJDR. 4:1768-1772.

Almoallim H, Aldahlawi S, Alqahtani E, Alqurashi S, Munishi A (2010). Difficulties facing first-year medical students at Umm Alqura University in Saudi Arabia. Eastern Mediterranean Health Journal. 16(12):1272-1277.
Al-Mously N, Salem R, Al-Hamdan N (2013). The impact of gender and English language on the academic performance of students: An experience from new Saudi medical school. J of Contemp Med Edu. 1(3):170-176.

Al-MulhimAA ,Elsharawy MA, Al Awad N (2012). The Influence of Gender on Saudi Students Performance in the Undergraduate Surgical Examination. Surgical Science. 3:206-209.

Chur-Hansen A, Vernon-Roberts J, Clark S (1997). Language background, English language proficiency and medical communication skills of medical students. Med Educ. 31(4):259-63.

Deepak KK, Al-umran KU, Al-heikh MH, Al-Rubaish A (2011). The influence of gender on Undergraduate Performance in Multiple Choice Testing in Clinical Disciplines at University of Dammam, Saudi Arabia. Al Ameen J Med Sci. 4(2): 123-130.

Dyrbye LN, Thomas MR, Shanafelt TD (2005). Medical student distress: causes, consequences, and proposed solution. Mayo Clin Proc. 80(12): 1613-22.

Frischenschlager O, Haidinger G, Mitterauer L (2005). Factors associated with academic success at Vienna Medical School: prospective survey. Croat Med J. 46(1):58-65.

Hall FR, Mikesell C Cranston P, Julian E, Elam C (2001). Longitudinal trends in the applicant pool for U.S. medical schools, 1974-1999. Acad Med. 76:829-34.

Hinkley RE (1997). Changes in profiles of students admitted to Florida allopathic medical schools 1990-1996. J Fla Med Assoc. 84(9):57882.

Ko SM, Kua EH, Fones CS (1999). Stress and the undergraduates. Singapore Med J. 40(10): 627-30.

Nuzhat A, Salem RO, Quadri MSA, Al-Hamdan N (2011). Learning style preferences of medical students: a single-institute experience from Saudi Arabia. International Journal of Medical Education. 2:70-73.

Salem RO, Al-Mously N, Nabil NM, Al-Zalabani AH, Al-Dhawi AF, AlHamdan N (2013). Academic and socio-demographic factors influencing students' performance in a new Saudi medical school. 35:83-89.

Salgar ST (2014). Stress in first year medical students. Int J of Biomed \&Adv Res. 5(1) 5-6.

Sawair FA, Baqain ZH, Al-Omari IKH, Wahab FK, Rajab LD (2009). Effect of gender on performance of undergraduate dental students at the university of Jordan , Amman. J Dent Educ. 73(11):1313-19.

Smith CK, Peterson DF, Degenhardt BF, Johnson JC (2007). Depression, anxiety, and perceived hassles among entering medical students. Psychol Health Med. 12(1):31-9. 